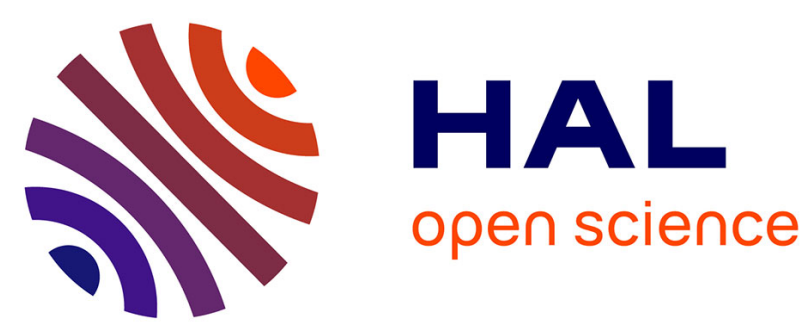

\title{
Rider weight consideration for observer design with an application to the estimation of the lateral motorcycle dynamics and rider's action
}

Pierre-Marie Damon, Dalil Ichalal, Lamri Nehaoua, Hichem Arioui, Saïd Mammar

\section{To cite this version:}

Pierre-Marie Damon, Dalil Ichalal, Lamri Nehaoua, Hichem Arioui, Saïd Mammar. Rider weight consideration for observer design with an application to the estimation of the lateral motorcycle dynamics and rider's action. 2017 IEEE International Conference on Systems, Man and Cybernetics (SMC 2017), Oct 2017, Banff, Canada. pp.3237-3242, 10.1109/SMC.2017.8123127 . hal-01760975

\section{HAL Id: hal-01760975 \\ https://hal.science/hal-01760975}

Submitted on 3 Oct 2019

HAL is a multi-disciplinary open access archive for the deposit and dissemination of scientific research documents, whether they are published or not. The documents may come from teaching and research institutions in France or abroad, or from public or private research centers.
L'archive ouverte pluridisciplinaire HAL, est destinée au dépôt et à la diffusion de documents scientifiques de niveau recherche, publiés ou non, émanant des établissements d'enseignement et de recherche français ou étrangers, des laboratoires publics ou privés. 


\title{
Rider weight consideration for observer design with an application to the estimation of the lateral motorcycle dynamics and rider's action
}

\author{
P-M Damon*, D. Ichalal*, L. Nehaoua*, H. Arioui* and S. Mammar* \\ *University of Evry Val d'Essonne, University of Paris-Saclay \\ IBISC Laboratory, Evry, France \\ Email: pierre-marie.damon@ibisc.univ-evry.fr
}

\begin{abstract}
This paper highlights the necessity of the rider weight consideration during observer's design for motorcycle dynamics estimation or control. It presents a novel approach using a linear parameter varying (LPV) model associated with the well-know Takagi-Sugeno (TS) methods to derive a robust observer regarding the rider weight uncertainty. Then the proposed solution is illustrated with an application to a proposed observer in our previous works by comparing results of estimation between a nominal, a heavier and a lighter rider. Finally, a complete simulation scenario shows the ability of the proposed method to estimate the lateral motorcycle dynamic states considering an uncertain rider weight.
\end{abstract}

\section{INTRODUCTION}

While the fatalities of the powered four wheeled vehicles (PFWV) users are constantly decreasing, the mortality of the motorcycle riders is still an important issue for many countries. By analogy with the PFWV market where the development of active and semi-active safety systems such as the anti-lock braking system (ABS), the electronic stability program (ESP), etc have largely contributed in the avoidance of many critical situations and in the reduction of crash severity, motorcycle makers have recently developed several similar systems. Among these systems one can cite: the antilock braking system (ABS) similar to the one for PFWV, the electronic stability control (ESC), the traction control system (TCS) or the motorcycle stability control (MSC) developed by Bosch.

In many countries PTWV are becoming the most common mean of transportation and are often dedicated to urban use. Currently there is a real challenge between manufacturers which try to sold new vehicles as cheap as possible to widen motorcycle user community. Emphasis on safety without significantly increase the selling price is a real challenge. That is why estimation and observation became major tools to make easier and cheaper the development of safety systems. They allow a reduction of the number of sensors and hence the cost is not so impact. Plenty of researches have addressed longitudinal and lateral PTWV dynamics estimation during the last years.

Among all the litterature, one can cite [1] and [2] where authors adressed longitudinal dynamic estimation whereas in [3], [4], [5], [6], [7] and [8] authors designed observers for lateral dynamics. An important difficulty during the observer's design for PTWV is the uncertainty of some parameters like the tire's properties (camber stiffness, cornering stiffness, radius of the wheel, etc.), the mass of fuel, the rider's properties, etc. The influence of the rider's morphology especially the mass, on the PTWV dynamics is very important and this paper proposed a novel approach to design a robust observer according to the rider mass uncertainty. A ratio between a nominal rider's weight and the real one is considered and the observer is synthesized with this ratio as a varying parameter which could be estimated with the longitudinal equation of PTWV motion. Finally, the Takagi-Sugeno approach is used to compute the observer's gains.

Before developing any active or semi-active safety system, the need of a mathematical model of the powered two wheeled vehicles (PWTV) is essential. Many works have addressed motorcycle modeling and for most of them longitudinal (inplane) dynamics and lateral (out-of-plane) dynamics model are clearly separated. Lateral PWT models have been largely studied in [9], [10], [11], [12] and many others. In the next section dealing with the lateral motorcycle modeling, the model presented in [12] is considered for the observer's design. This model is very similar to the one used in the well-known multi-body simulator BikeSim which represents the motorcycle as a set of 9 bodies and allows 16 degree of freedom. Then the model is then linearized around straight trajectory to facilitate observer's synthesis. Finally, it allows 4 degrees of freedom (DOF): the yaw, the roll, the steering and the lateral slip.

This paper is organized as follows. Section 2 presents motivations and states the problem. The model of the motorcycle is introduced in section 3 whereas in section 4 , we recall the main steps of the design of the observer which is used to illustrate the proposed approach. Simulation results are discussed in section 5 and finally concluding remarks will be provided in section 7 .

\section{Motivation}

When studying motorcycles, the ratio between rider and vehicle mass cannot be neglected and it highly depends of the vehicle's category. Indeed, among the lightest PWTV the most common are the small scooters weighting around $80 \mathrm{~kg}$ whereas some big cruisers or big touring bikes weight more 
than $400 \mathrm{~kg}$. Very few investigations deal with the global motorcycle market statistics especially about the vehicle's features (weight, dimensions, etc). Nevertheless, the small scooters and the heavy motorcycles are not the most common PWTV, there are lot of intermediate categories of bikes: sport, racing, roadster, big scooter, ... Considering all this vehicles features, let us consider an average motorcycle's mass around $180 \mathrm{~kg}$. In [13] the author presents an anthropometric study concerning the morphology of motorcycle's rider in UK and highlights the average rider's profile which weights around 78 $\mathrm{kg}$. Taking into account the rider's equipment: helmet, gloves, boots, motorcycle jacket, let us increase the average rider's mass to $85 \mathrm{~kg}$ which leads to the ratio for a single rider without passenger $M_{\text {rider }} / M_{\text {vehicle }}=0.47$. Unlike motorcycle, the average mass for all the car sold in EU during 2015 is around $1400 \mathrm{~kg}$ [14] and considering the same single driver morphology the ratio becomes $M_{\text {driver }} / M_{\text {vehicle }}=0.06$. Moreover, the most of PWTV are approved for a driver with a passenger and additional masses (payloads, bags, etc) for a maximum mass which may be less than the gross vehicle weight rating (GVWR) specified on the vehicle registration document. By analogy with motorcycles, it depends on the car's category but PFWV are often approved for one driver with 4 passengers and payloads. This comparison clearly shows the necessity to consider the different rider morphologies especially for PTWV purposes.

Previous studies have ever deal with the rider's weight uncertainty like in [15] where the author studies the influence of the rider's properties on the PTWV stability especially on the weave mode. In [16] or [17], authors used a common method to include rider mass uncertainty which is taken into consideration as an uncertain state matrix and input vector such as in the state space representation $A=A_{\text {nominal }}+\Delta A$ and $B=B_{\text {nominal }}+\Delta B$, with $\Delta A$ and $\Delta B$ containing the uncertainties regarding the nominal case. Then, the robust techniques such as $L_{2}$-gain are used to minimize the transfer of the term due to the uncertainties on the estimation errors. Nevertheless, such a method always leads to a LMI formulation with a parameter to be minimized and sometimes no solution exists to design a robust observer. The following work proposes a novel approach to get around the problem of the insolvable LMIs.

The development of the proposed method is based on our previous work [4]. In this paper, an observer is synthesized to estimate the lateral motorcycle dynamics states and the rider's action on the handlebar. The observer's design is derived from a LPV model depending of the longitudinal velocity $v_{x}$. The model is computed since the nominal case which means for a nominal rider and doesn't take into account any uncertainty. Then the convergence study is based on the Lyapunov theory associated with LMI tools and $L_{2}$-gain to guarantee boundlessness of the state estimation error. Notice that here the $L_{2}$-gain technic is not used to handle the model's uncertainties but in order to guarantee the error convergence of the augmented system. In [4] the effectiveness of the proposed nominal observer has been tested for a simulation scenario with two successive double lane changes (DLC) at variable speed. In addition, a simple DLC scenario at constant speed $100 \mathrm{~km} / \mathrm{h}$ is simulated with the nominal observer and then with rider weight's uncertainty in the model. For the considered scenario the estimation error due to this uncertainty is acceptable.

However a deeper investigation has shown that for more complex scenarios with highly variable forward speed, the proposed observer is not enough robust regarding the rider's weight uncertainty. For example, let us consider a road circuit simulation without any limitation on the forward speed, the observer is initially derived with the nominal driver weight of $85 \mathrm{~kg}$ and then the rider's weight parameter in the model is increased of $20 \mathrm{~kg}$ to $105 \mathrm{~kg}$. Figure 12 clearly shows the error introduced by this uncertainty and the necessity to consider it during the observer's design. Notice that introducing the rider's weight uncertainty in a state matrix and input vector respectively $\Delta A$ and $\Delta B$ lead to unsolvable LMIs. That is why this paper proposed another approach to deal with this issue.

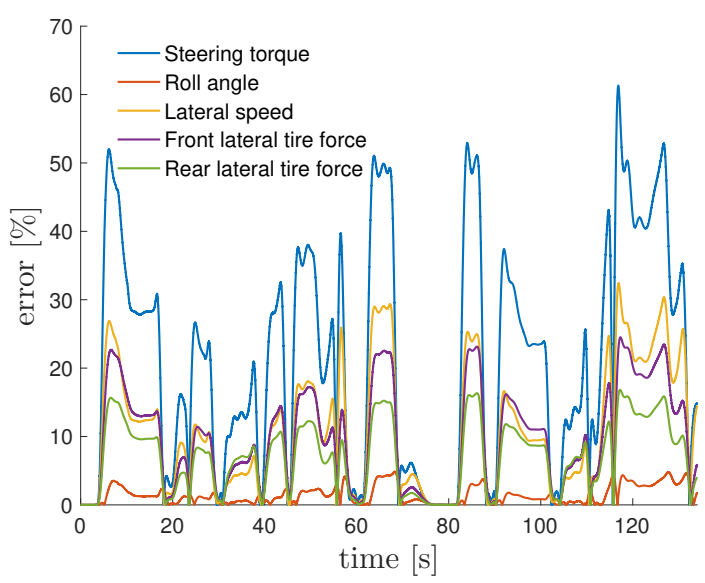

Fig. 1. State's estimation error (\%)

\section{MOTORCYCLE DYNAMICS}

\section{A. Rider mass property and its estimation}

This section aims to describe how the rider mass uncertainty is considered. The lateral motorcycle model which is presented below is based on a multi-body model of the whole motorcycle and rider who is considered as a set of two rigid bodies composed of an upper and a lower bodies. To facilitate the rider mass consideration during modeling, let us consider the following assumptions: lower and upper gravity center positions are considered constant and the mass is equally distributed between the upper and lower body according to the initial repartition $M_{\text {rider }_{\text {upper }}} / M_{\text {rider }_{\text {lower }}}=1.68$. The rider weight uncertainty problem is addressed by considering a weight ratio $r_{w}$ which represents the ratio between nominal rider weight used to design the nominal model or observer and the real rider one. $r_{w}>1$ and $r_{w}<1$ means respectively that the real rider is heavier or lighter than the nominal one. This ratio not only affects the rider weight parameter but also 
the inertial properties which linearly depends of the body's mass. Hence, taking into account the rider's weight uncertainty imply of course to change the corresponding rider weights for the upper body and the lower body by multiplying with the rider's weight ratio $r_{w}$ but also to modify the inertia matrix associated to the two rider's bodies. Let us remind that each term $I_{i j} \quad i, j=x, y, z$ of an inertia tensor of a rigid body depends linearly on the mass. So the upper and lower rider inertia tensors will be also multiplied by $r_{w}$.

The obvious way to estimate the rider mass $M$ and to deduce the ratio $r_{w}$ is to use the longitudinal model of the motorcycle. Indeed, under some assumptions the mass can be identified during acceleration at low speed. Longitudinal model of the motorcycle is described by:

$$
\left\{\begin{array}{l}
M \dot{v}_{x}=F_{x f}+F_{x r}-F_{r e s} \\
i_{y f} \dot{\omega}_{f}=-R_{f} F_{x f}+T_{f} \\
i_{y r} \dot{\omega}_{r}=-R_{r} F_{x r}+T_{r}
\end{array}\right.
$$

with $M$ the global mass including rider and PTWV masses, $\dot{v}_{x}$ the longitudinal acceleration, $R_{i}$ the radius of the wheels, $F_{x i}$ the longitudinal tire forces, $i_{y i}$ the wheel inertia, $\dot{\omega}_{i}$ the wheel angular accelerations and $T_{i}$ the driving or braking torques. $F_{\text {res }}$ is the resistant forces such as $F_{\text {res }}=F_{\text {rolling }}\left(v_{x}\right)+$ $F_{\text {aero }}\left(v_{x}^{2}\right)+F_{\text {slope }}$

For low speed model during acceleration $\left(\dot{v}_{x} \neq 0\right)$ on flat road, the longitudinal equation of motion can be simplified according to the following assumptions: there is no driving/braking torque applied on the front wheel $T_{f}=0$, the rolling forces $F_{\text {rolling }}\left(v_{x}\right)$, the aerodynamic forces $F_{\text {aero }}\left(v_{x}^{2}\right)$ and the force due to the road slope $F_{\text {slope }}$ are neglected. It comes :

$$
M=\frac{1}{\dot{v}_{x}}\left(-\frac{i_{y f}}{r_{f}} \dot{\omega}_{f}-\frac{i_{y r}}{r_{r}} \dot{\omega}_{r}+\frac{1}{r_{r}} T_{r}\right)
$$

Since the ABS equippes the most part of the new vehicles, the wheel speed sensors included in the system allow to measure the angular velocity of the front and rear wheels respectively $\omega_{f}$ and $\omega_{r}$. With the recent differentiation techniques [18] it is easy to obtain a reliable estimation of the time derivatives of the wheel speed. In addition, the growing market of low cost sensors and the development of the embedded electronics gives the opportunity of the motorcycle makers to equip new vehicles with other kind of sensors like inertial measurement unit (IMU) or with simple accelerometers (in both cases, the sensor provides a measure of the longitudinal acceleration $a_{x}$ ). Moreover, the democratization of the injection engines in the motorcycle field enables to know the driving torque in real time. Indeed, the use of the injection is based on an engine map giving the outgoing torque on the driving shaft. With the features of the transmission mechanism especially the ratio, the driving torque on the rear wheel $T_{r}$ is considered known. Hence, the equation (2) allows us to estimate the rider's mass $M_{\text {rider }}$ knowing the vehicle mass $M_{v e h}$ with the equation $M_{\text {rider }}=M-M_{v e h}$.

\section{B. Lateral Model of motorcycle}

The present section is based on our previous works. The main steps of the model's derivation are summarized here but for more detail please refer to [19] and [4]. Lateral dynamics of the motorcycle involves the roll inclination, the yaw rotation, and the steering and lateral motions of the bike. The coupling between the longitudinal and lateral motions is materialized by considering a variable longitudinal velocity that appears in the lateral dynamics. The multi-body model given in [19] is linearized around the straight-running trim trajectory and includes the rider mass uncertainty as a second varying parameter, it can be expressed by the following state space representation:

$$
\left\{\begin{aligned}
\dot{\tilde{x}} & =\tilde{A}\left(v_{x}, r_{w}\right) \tilde{x}+\tilde{B}\left(r_{w}\right) \tau \\
\tilde{y} & =\tilde{C} x
\end{aligned}\right.
$$

where $\tilde{x}=\left[\phi, \delta, v_{y}, \dot{\psi}, \dot{\phi}, \dot{\delta}, F_{y f}, F_{y r}\right]^{T}$ denotes the state vector and $\tau$ is the rider's torque. $\tilde{A}\left(v_{x}, r_{w}\right)$ and $\tilde{B}\left(r_{w}\right)$ are time-varying state matrices and input vector related to the forward velocity $v_{x}$ and the driver weight's ratio $r_{w}$. To guarantee observability and observer design conditions the needed measurements are the yaw rate $\dot{\psi}$ and roll rate $\dot{\phi}$ (both are given by the central unit); the steering angle $\delta$ and steering rate $\dot{\delta}$ which may be obtained from an optical encoder. Finally, with the appropriate output matrix $\tilde{C}$ it comes $\tilde{y}=[\delta, \dot{\psi}, \dot{\phi}, \dot{\delta}]^{T}$. Note that $\tilde{C}$ is not time-varying. For more details about the matrices and the vector please refer to Appendix.

According to the unmeasurability of the rider's torque $\tau$ and the existence of its first time derivative $\dot{\tau}$ which is always satisfied because the rider's torque is naturally bounded and continuous, the system is augmented as it follows :

$$
\left\{\begin{aligned}
\dot{x} & =\left[\begin{array}{cc}
\tilde{A}\left(v_{x}, r_{w}\right) & \tilde{B}\left(r_{w}\right) \\
0 & 0
\end{array}\right] x+\left[\begin{array}{l}
0 \\
1
\end{array}\right] \dot{\tau} \\
y & =\left[\begin{array}{ll}
\tilde{C} & 0
\end{array}\right] x
\end{aligned}\right.
$$

with $x=[\tilde{x}, \tau]^{T}$ and $y=\tilde{y}$ which respectively denote the augmented state vector and the vector of the measures. Note that the system as expressed in (4) is an exact form considering that $\dot{\tau}$ exists.

For the next sections let us consider the following notations:

$$
\begin{aligned}
A\left(v_{x}, r_{w}\right) & =\left[\begin{array}{cc}
\tilde{A}\left(v_{x}, r_{w}\right) & \tilde{B}\left(r_{w}\right) \\
0 & 0
\end{array}\right], C=\left[\begin{array}{ll}
\tilde{C} & 0
\end{array}\right], \\
F & =\left[\begin{array}{l}
0 \\
1
\end{array}\right], f=\dot{\tau}
\end{aligned}
$$

\section{Exact T-S model of the augmented model}

In order to express the LPV model (4) in T-S fuzzy structure, let us consider the non-linearities $v_{x}$ and $r_{w}$.

By following the well-known sector non-linearity approach [20], The state equation of (4) can be exactly expressed as follows:

$$
\dot{x}=\sum_{i=1}^{4} \mu_{i}\left(v_{x}, r_{w}\right) A_{i} x+F f
$$


Note that there is two non-linearities that's why the system is described with $2^{2}=4$ sub-models. The variables $\mu_{i}($.$) are$ called membership or weighting functions and must satisfy the following convex sum property:

$$
\left\{\begin{array}{l}
\sum_{i=1}^{4} \mu_{i}\left(v_{x}, r_{w}\right)=1 \\
0 \leq \mu_{i}\left(v_{x}, r_{w}\right) \leq 1
\end{array}\right.
$$

Let us introduce the functions $h_{i}() \quad i=1,. . ., 4$ such as:

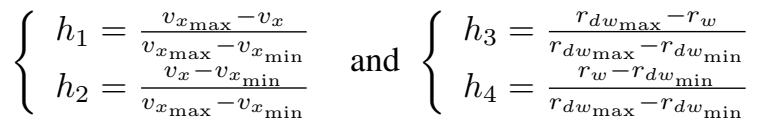

Then the $\mu_{i}($.$) functions are computed as it follows:$

$$
\left\{\begin{array} { l } 
{ \mu _ { 1 } = h _ { 1 } \cdot h _ { 3 } } \\
{ \mu _ { 2 } = h _ { 2 } \cdot h _ { 3 } }
\end{array} \text { and } \left\{\begin{array}{l}
\mu_{3}=h_{1} \cdot h_{4} \\
\mu_{4}=h_{2} \cdot h_{4}
\end{array}\right.\right.
$$

Finally, the augmented model (6) expressed in exact TS form allows to design a Luenberger observer to estimate the vehicle lateral dynamics and the rider's torque.

\section{OBSERVER DESIGN}

In this section let us remind the main steps of the observer's design. Consider the full state-space representation in TS form:

$$
\left\{\begin{aligned}
\dot{x} & =\sum_{i=1}^{4} \mu_{i}\left(v_{x}, r_{w}\right) A_{i} x+F f \\
y & =C x
\end{aligned}\right.
$$

For the observer design the two non-linearities $v_{x}$ and $r_{w}$ are considered measured. The measure of $r_{w}$ have been discussed in the previous section.

Consider the well-known Luenberger observer in TS form:

$$
\left\{\begin{aligned}
\dot{\hat{x}} & =\sum_{i=1}^{4} \mu_{i}\left(v_{x}, r_{w}\right)\left(A_{i} \hat{x}+L_{i}(y-\hat{y})\right) \\
\hat{y} & =C \hat{x}
\end{aligned}\right.
$$

with $L_{i}$ the observer gain matrices which ensure error convergence. The estimated states and output vector are respectively denoted $\hat{x}$ and $\hat{y}$. Now consider the state estimation error as follows:

$$
e=x-\hat{x}
$$

Finally, the dynamic of the error can be expressed as it follows:

$$
\dot{e}=\sum_{i=1}^{4} \mu_{i}\left(v_{x}, r_{w}\right) \mathcal{A}_{i} e+F f
$$

with $\mathcal{A}_{i}=A_{i}-L_{i} C$.

Let us consider the Lyapunov function $V$ with the symmetric definite positive matrix $X$ such that:

$$
V=e^{T} X e, \quad X=X^{T}>0
$$

whose time derivatives $\dot{V}$ leads to:

$$
\begin{aligned}
& \dot{V}=e^{T} \sum_{i=1}^{4} \mu_{i}\left(v_{x}, r_{w}\right)\left(\mathcal{A}_{i}^{T} X+X \mathcal{A}_{i}\right) \quad e+f^{T} F^{T} X e \\
& +\quad e^{T} X F f(14)
\end{aligned}
$$

To attenuate the effect of the perturbation $f$ on the estimation error $e$ let us define the $L_{2}$-gain as the quantity:

$$
\sup _{\|f\|_{2} \neq 0} \frac{\|e\|_{2}}{\|f\|_{2}} \leq \gamma^{2}
$$

with $\gamma$ a positive scalar and $\|.\|_{2}$ the $L_{2}$-norm which for a vector $z(t)$ is given by:

$$
\|z(t)\|_{2}=\left(\int_{0}^{\infty} z^{T}(t) z(t) d t\right)^{1 / 2}
$$

The $L_{2}$-gain leads to the inequality:

$$
e^{T} e-\gamma^{2} f^{T} f<0
$$

Considering $\dot{V}<0$ it follows:

$$
\dot{V}+e^{T} e-\gamma^{2} f^{T} f<0
$$

By exploiting the expression of $\dot{V}$, inequality (18) can be expressed in matrix form as follows:

$$
\left[\begin{array}{l}
e \\
f
\end{array}\right]^{T}\left[\begin{array}{cc}
\Theta & X F \\
F^{T} X & -\gamma^{2}
\end{array}\right]\left[\begin{array}{l}
e \\
f
\end{array}\right]<0
$$

with $\Theta=\sum_{i=1}^{4} \mu_{i}\left(v_{x}, r_{w}\right)\left(\mathcal{A}_{i}^{T} X+X \mathcal{A}_{i}\right)+I$

Let us consider the following change of variables $\bar{L}_{i}=$ $X L_{i}, A_{i}=\mathcal{A}_{i}+L_{i} C$ and $\bar{\gamma}=\gamma^{2}$. Since the weighting functions satisfy the convex sum property, sufficient conditions ensuring the convergence of estimation error are obtained as follows:

$$
\left[\begin{array}{cc}
A_{i}^{T} X+X A_{i}-\bar{L}_{i} C-C^{T} \bar{L}_{i}^{T}+I & X F \\
F^{T} X & -\bar{\gamma}
\end{array}\right]<0, \quad i=1, \ldots, 4
$$

Finally, given a scalar $\gamma$, if there exists a symmetric and positive definite matrix $\mathrm{X}$ and matrices $\bar{L}_{i}, i=1, \ldots, r$ such that the LMI (20) is satisfied, then the error is stable and the transfer from the perturbation $f$ to the estimation error $e$ is bounded by $\gamma$. Note that the observer gain matrix $L$ is obtained by:

$$
L=\sum_{i=1}^{4} \mu_{i}\left(v_{x}, r_{w}\right) X^{-1} \bar{L}_{i}
$$

In practice to get better performances of the observer it is possible to transform the previous LMI (20) in an optimization problem by considering $\gamma$ as a variable parameter of optimization.

\section{Simulation Results}

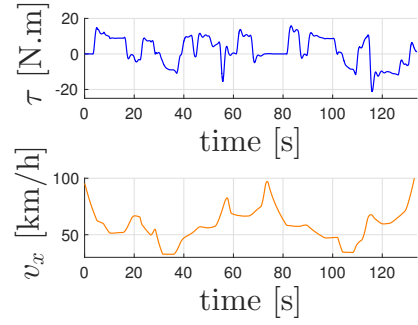

(a) Longitudinal speed

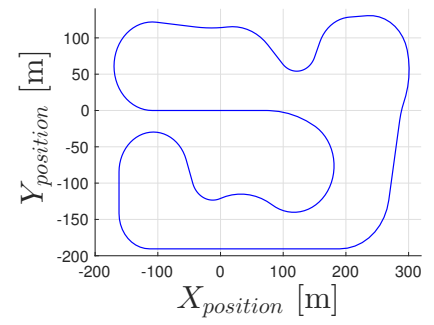

(b) Vehicle's trajectory
Fig. 2. Simulation scenario 
This section aims to show the ability of the proposed solution to robustly estimate the lateral motorcycle dynamics states according to the rider's mass variations. The simulation is performed on a complete riding scenario without any restriction especially on the forward speed and the trajectory. A first simulation is performed with a nominal rider weighting $85 \mathrm{~kg}$ leading to a ratio $r_{w}=1$ and two others with a lighter and a heavier rider respectively weighting $65 \mathrm{~kg}$ and $105 \mathrm{~kg}$ (more or less $20 \mathrm{~kg}$ around the nominal case) and leading respectively to $r_{w}=0.76$ and $r_{w}=1.24$. The two nonnominal riders aims to represent the morphology inequalities among the motorcycle's users.

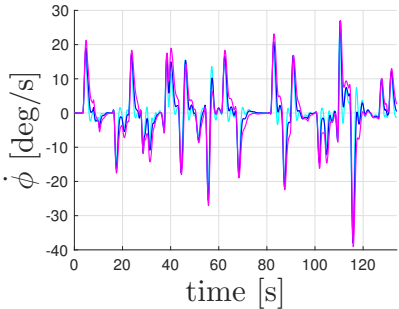

(a) Measured roll rate for light (cyan), nominal (blue) and heavy (magenta) rider

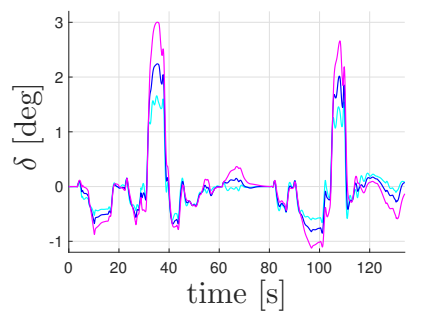

(c) Measured steering angle for light (cyan), nominal (blue) and heavy (magenta) rider

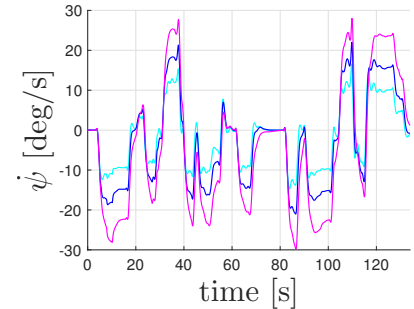

(b) Measured yaw rate for light (cyan), nominal (blue) and heavy (magenta) rider

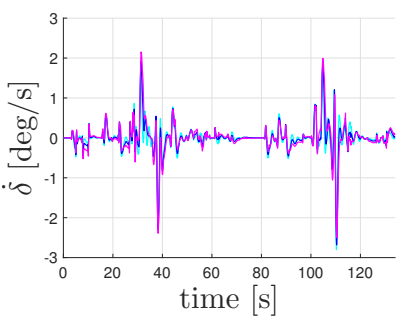

(d) Measured steering rate for light (cyan), nominal (blue) and heavy (magenta) rider

Fig. 3. Measured states

The performed scenario is exactly the same for the three simulations and directly comes from the well-known simulator BikeSim. This simulator allows to record the rider's torque applied on the handlebar and the forward speed along the circuit which are the input of our model. For the sake of clarity the figure 2 introduced only the rider's torque and the velocity for the nominal rider and then the results compare the different cases.

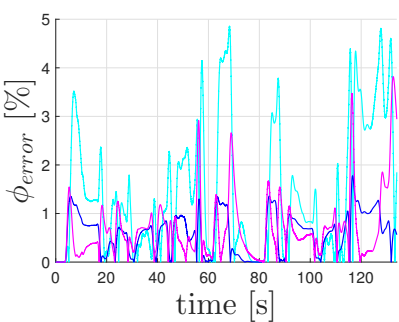

(a) Roll angle estimation error for light (cyan), nominal (blue) and heavy (magenta) rider

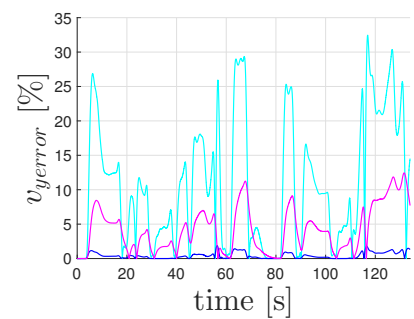

(b) Lateral velocity estimation error for light (cyan), nominal (blue) and heavy (magenta) rider

Fig. 4. State's estimation with the nominal observer

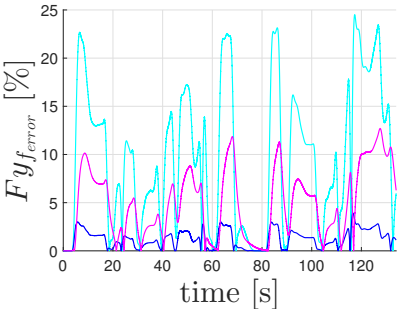

(c) Front lateral tire force estimation error for light (cyan), nominal (blue) and heavy (magenta) rider

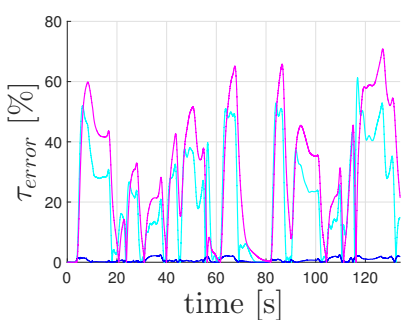

(e) Rider's torque estimation error for light (cyan), nominal (blue) and heavy (magenta) rider

Fig. 4. State's estimation with the nominal observer

Figure 3 shows the measured states along the track for the three different riders. Note that the same colors are adopted for the next graphics: cyan, blue and magenta respectively represent the light, the nominal and the heavy rider. As discussed previously, an IMU allows us to measure the roll and yaw rate whereas an optical encoder installed on the steering column gives a measure of the steering angle and rate.

Figure 4 represents the estimation's error with the nominal observer designed with $r_{w}=1$ and the different riders. It is clear that the rider mass uncertainty leads to important error without any consideration during the observer design. Please note that the error is only bounded with ISS property that is why even with the nominal observer and the nominal rider the state estimation is non-zero.

Figure 5 illustrates the ability of the proposed method to robustly estimate the dynamics states with rider mass uncertainty. Compared to figure 4 where the estimation error is sometimes more than $60 \%$ for the rider's torque with the proposed approach the estimation error is inferior to $5 \%$ for all the states.

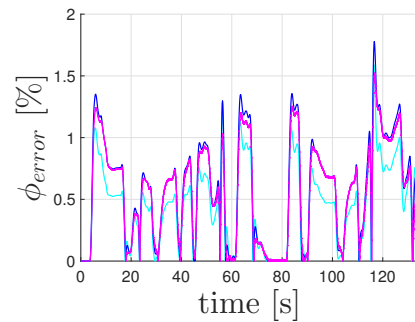

(f) Roll angle estimation error for light (cyan), nominal (blue) and heavy (magenta) rider

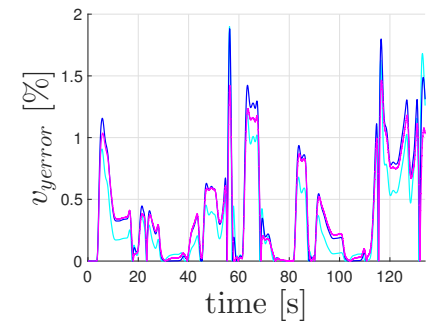

(g) Lateral velocity estimation error for light (cyan), nominal (blue) and heavy (magenta) rider
Fig. 5. State's estimation with the proposed observer 


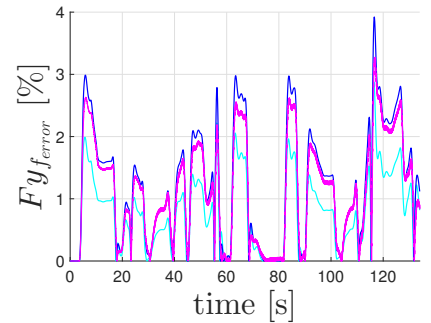

(h) Front lateral tire force estimation error for light (cyan), nominal (blue) and heavy (magenta) rider

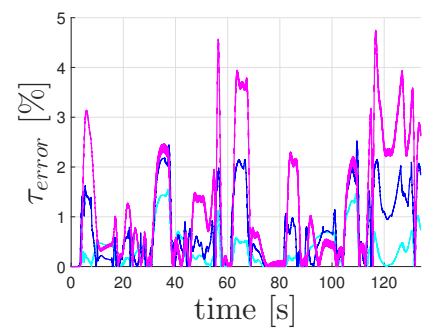

(j) Rider's torque estimation error for light (cyan), nominal (blue) and heavy (magenta) rider

Fig. 5. State's estimation with the proposed observer

\section{CONCLUSION}

The present work clearly shows the necessity of the rider's mass consideration during observer design and more generally during the development of advanced rider assistance systems (ARAS) for PTWV. The proposed solution uses a novel approach using the well-known sector non-linearity technic regarding the rider's weight uncertainty. Then the method is explained during the design of an observer for lateral dynamics and rider's action estimation. The considered observer have been already introduced in [4] for a nominal case and the main steps of the synthesis are remind. Finally, a complete circuit simulation without any restriction on the motorcycle dynamics demonstrates the ability of the proposed approach to robustly estimate the lateral dynamics states and the rider's torque with different rider's weight.

\section{APPENDIX}

\begin{tabular}{ll}
\hline Variables, matrices and notations \\
\hline$\dot{x}, \ddot{x}$ & time derivatives of the variable $x$ \\
$\hat{x}$ & estimate of a variable $x$ \\
$x^{T}$ & transpose of vector or matrix $x$ \\
$x_{f}, x_{r}$ & denotes front and rear \\
$v_{x}, v_{y}$ & longitudinal and lateral vehicle speeds \\
$\omega_{f}, \omega_{r}$ & angular wheel speeds \\
$\phi, \psi, \delta$ & roll, yaw and steer angles \\
$\tau$ & rider's torque \\
$M$ & global mass (rider and vehicle) \\
$r_{w}$ & rider's weight ratio \\
$F_{x_{f}}, F_{x_{r}}$ & longitudinal tire forces \\
$F_{y_{f}}, F_{y_{r}}$ & lateral tire forces \\
$\tilde{A}, A$ & state matrices \\
$\tilde{B}, B$ & input vectors \\
$C$ & observation matrix \\
$F$ & perturbation matrix
\end{tabular}

\section{REFERENCES}

[1] H. Dabladji, D. Ichalal, H. Arioui, and S. Mammar, "On the estimation of longitudinal dynamics of powered two-wheeled vehicles," in European Control Conference, 2015.

[2] M. Corno, G. Panzani, and S. M. Savaresi, "Traction-control-oriented state estimation for motorcycles," Control Systems Technology, IEEE Transactions on, vol. 21, no. 6, pp. 2400-2407, 2013.

[3] L. Nehaoua, D. Ichalal, H. Arioui, S. Mammar, and L. Fridman, "An unknown-input HOSM approach to estimate lean and steering motorcycle dynamics," IEEE Transactions on Vehicular Technology, vol. 63, no. 7, pp. 3116-3127, Sept 2014.

[4] P. Damon, H. Dabladji, D. Ichalal, L. Nehaoua, and H. Arioui, "Estimation of lateral motorcycle dynamics and rider action with luenberger observer," pp. 2392-2397, 2016.

[5] P. M. Damon, H. Dabladji, D. Ichalal, L. Nehaoua, H. Arioui, and S. Mammar, "Lateral motorcycle dynamics and rider action estimation: An lpv unknown input observer approach," in 2016 IEEE Conference on Control Applications (CCA), Sept 2016, pp. 711-716.

[6] D. Ichalal, H. Dabladji, H. Arioui, S. Mammar, and L. Nehaoua, "Observer design for motorcycle lean and steering dynamics estimation: A Takagi-Sugeno approach," in American Control Conference (ACC), June 2013, pp. 5654-5659.

[7] I. Boniolo, S. M. Savaresi, and M. Tanelli, "Lean angle estimation in two-wheeled vehicles with a reduced sensor configuration," in 2012 IEEE International Symposium on Circuits and Systems, May 2012, pp. $2573-2576$

[8] A. Teerhuis and S. Jansen, "Motorcycle state estimation for lateral dynamics," Vehicle System Dynamics, vol. 50, no. 8, pp. 1261-1276, 2012.

[9] R. S. Sharp, "The stability and control of motorcycles," Journal of Mechanical Engineering Science, vol. 13, no. 5, pp. 316-329, 1971.

[10] R. Sharp, S. Evangelou, and D. J. Limebeer, "Advances in the modelling of motorcycle dynamics," Multibody system dynamics, vol. 12, no. 3, pp. 251-283, 2004

[11] V. Cossalter, Motorcycle dynamics. Lulu, 2006.

[12] L. Nehaoua, H. Arioui, N. Seguy, and S. Mammar, "Dynamic modeling of a two wheeled vehicle: Jourdain formalism," Vehicle System Dynamics, 2013.

[13] S. Robertson and A. Minter, "A study of some anthropometric characteristics of motorcycle riders," Applied Ergonomics, vol. 27, no. 4, pp. $223-229,1996$.

[14] T. I. C. o. c. T. icct, "European vehicle market statistics 2016/17," icct, The Internal Council on clean Transportation, Tech. Rep., 2017.

[15] M. Massaro, R. Lot, V. Cossalter, J. Brendelson, and J. Sadauckas, "Numerical and experimental investigation of passive rider effects on motorcycle weave," Journal of Vehicle System Dynamics, vol. 50, pp. 215-227, March 2012.

[16] C. Chenane, D. Ichalal, H. Arioui, and S. Mammar, "Proportional two integral (p2i) observer synthesis for single track vehicle," pp. 1530-1535, July 2012.

[17] H. Dabladji, D. Ichalal, H. Arioui, and S. Mammar, "Unknown-input observer design for motorcycle lateral dynamics: Ts approach," Control Engineering Practice, vol. 54, pp. 12-26, 2016.

[18] M. Fliess, C. Join, and H. Sira-Ramirez, "Non-linear estimation is easy," International Journal of Modelling, Identification and Control, vol. 4, no. 1, pp. 12-27, Jan. 2008.

[19] L. Nehaoua, L. Nouvellière, and S. Mammar, "Dynamics modeling of a two-wheeled vehicle using Jourdain's principle," in 19th Mediterranean Conference on Control Automation (MED),, June 2011, pp. 1088-1093.

[20] K. Tanaka and H. Wang, Fuzzy Control Systems Design and Analysis: A Linear Matrix Inequality Approach. John Wiley and Sons, 2001. 